\title{
An 8-Week Group Cognitive Behavioral Therapy Intervention for Mobile Dependence
}

\author{
Ting Li, Lixia Cui*, Shu Ma, Shuang Zhang, Jie Zheng, Jing Xiao*, Qin Zhang \\ Beijing Key Laboratory of Learning and Cognition and School of Psychology, Capital Normal University, Beijing, China \\ Email: *cuilixia@cnu.edu.cn, ${ }^{*}$ xiaojingcnu@163.com
}

How to cite this paper: Li, T., Cui, L. X., Ma, S., Zhang, S., Zheng, J., Xiao, J., \& Zhang, Q. (2018). An 8-Week Group Cognitive Behavioral Therapy Intervention for Mobile Dependence. Psychology, 9, 2031-2043. https://doi.org/10.4236/psych.2018.98116

Received: July 3, 2018

Accepted: August 5, 2018

Published: August 8, 2018

Copyright $(9) 2018$ by authors and Scientific Research Publishing Inc. This work is licensed under the Creative Commons Attribution International License (CC BY 4.0).

http://creativecommons.org/licenses/by/4.0/

\begin{abstract}
In China, college students with mobile phone dependence are more and more common, which is accompanied by many psychological problems. We developed a group cognitive therapy (CBT) program for mobile phone dependence and studied its effects in Chinese college students. Forty-two Chinese college students, whose scores in the revised Chinese Test of Mobile Phone Dependence (C-TMD) were 37 to 42 points, were assigned to two conditions at a random: the group CBT intervention and the wait-list control condition. The result showed that the CBT group demonstrated significantly more decrease in measures of the C-TMD and Simplified Symptom Checklist (SSCL) than the wait-list controls at post-test and two-month follow-up.
\end{abstract}

\section{Keywords}

Mobile Phone Dependence, CBT-IA, Group CBT, C-TMD

\section{Introduction}

By the early 21st century, mobile phones had become ubiquitous in everyday life in many countries, but researchers have found that this new technology is associated with many problems. Excessive mobile phone use may be a health-compromising behavior, like drinking alcohol or smoking (Koivusilta, Lintonen, \& Rimpelä, 2003; Leena, Tomi, \& Arja, 2005). Self-symptom reports show that mobile phone use is generally associated with headaches, earache, fever, and other symptoms (Korpinen, \& Pääkkönen, 2009), and sometimes leads to inattention and fatigue (Johansson, Nordin, Heiden, \& Sandström, 2010), even predicts negatively academic performance of college students (Lepp, Barkley, \& Karpinski, 2015). Mobile phone use in a variety of settings will lead to serious consequences, and it is banned in some jurisdictions (Bianchi \& Phillips, 2005). Thomée, Eklöf, Gus- 
tafsson, Nilsson, and Hagberg (2007) found that a large number of mobile phone hours spent on mobile phones at baseline was associated with an increased risk of reporting prolonged stress and symptoms of depression at one-year follow-up. Frequent mobile phone use won't only take more money and time, but also produce negative emotions for young adults (Chen, Yan, Tang, Yang, Xie, \& $\mathrm{He}, 2016)$, which is significantly associated with depression among 13 - 20 years old (Sanchez-Martinez \& Otero, 2009). Thus public health prevention should focus on providing information and advice for young people to help them curb mobile phone use (Thomée, Härenstam, \& Hagberg, 2011).

In recent years, some research has been done on the characteristics and nature of mobile phone dependence. Thinking of mobile phone dependence as a kind of techno-stress (Brod, 1984), researchers defined mobile phone dependence by two factors: excessive mobile phone use and unreasonable use in public places despite it being a nuisance (Toda, Monden, Kubo, \& Morimoto, 2006). One study on mobile phone dependence illustrated that we could associate actual use of the mobile phone (number and duration of the calls made in one day, number of text message system sent daily) with high urgency and low perseverance (Billieux, Linden, \& Rochat, 2008). Walsh, White, Cox , and Young (2011) posit that different psychological process underpin the frequency and involvement of mobile phone use, such as self-identify, in-group norms and self-motivations. People with higher attachment anxiety use mobile phone more frequently (Konok, Gigler, Bereckzy, \& Ádám, 2016).

Chóliz (2012) reported that teenagers who used mobile phone excessively met the characteristic criteria of substance use disorders according to the DSM-5 (DSM-V; American Psychiatric Association, 2013): 1) Excessive use, they send numerous messages and frequently make telephone calls with a high economic cost; 2) Problems, some problems of excessive use are related to parent-child relationships in particular; 3) Interference, cell phone use interferes with other school or personal activities; 4) Frequency, they increase the frequency of use and replace functioning devices with newer models to obtain the same level of satisfaction; 5) Mood, their mood will be affected without using mobile phone use. Chóliz (2012) designed the test of mobile phone dependence (TMD) to evaluate mobile phone dependence.

While the harm caused by mobile phone dependence has been well established, there have been few studies on interventions for mobile dependence despite a pressing need to develop and implement effective prevention. Some researchers have found that Internet addiction is related to impulsive control disorder (e.g., Aboujaoude, Koran, Gamel, Large, \& Serpe, 2006; Beard \& Wolf, 2001; Block, 2008; Shapira et al., 2003; Young, 1998), the frequency of mobile dependence is associated with impulsivity (Mei, Chai, Wang, Ng, Ungvari, \& Xiang, 2018). CBT is thought to be an effective treatment of impulsive control disorder (Hucker, 1995). In addition, CBT has also been shown to be effective in treating substance abuse, mood disorders, and eating disorders (Beck, 1976; Beck, Wright, Newman, \& Liese, 1993). One research indicates that cognitive 
distortion is the mediator of Pathological Internet Use (PIU) (Lu \& Yeo, 2015). On the basis of similarities between Internet addiction and impulsive control disorders, Young (2013) designed the cognitive-behavioral therapy for Internet addiction (CBT-IA); the results suggested that CBT-IA effectively ameliorated symptoms associated with Internet addiction, and the effects could be maintained for six months. The literature shows that there are many similarities between mobile phone dependence and Internet dependence. First, Internet dependence and mobile phone dependence have many of the same features (Tian, Bian, Han, Gao, \& Wang, 2017; Chóliz, 2012; Mei et al., 2018; Aboujaoude et al., 2006): 1) both mobile phone and Internet dependence are a kind of excessive use; 2) both are related to impulse-control disorders; 3) both are accompanied by compulsive use; 4) dependents will increase the time spent on the activity to get the same level of satisfaction in reality; 5) both need some economic input; 6) both will cause negative consequences in daily life (family, school, occupation, or social level); 7) users regard it as a means of regulating emotions; 8) both have symptoms of withdrawal; 9) users are more tolerant of their excessive use of behavior, and believe in their rationality; 10) both are daily necessities. Apart from these same features, the specificity of mobile phone dependence is still manifested in convenience, universality, simplicity, and mobile phone dependence is more common and serious than Internet dependence (Chen et al., 2016; China Internet Network Information Center [CNNIC], 2015).

Because of the similarities and difference between mobile phone dependence and Internet dependence, we designed a group CBT program for mobile phone dependence, on the basis of cognitive-behavioral therapy for internet addiction (CBT-IA), and we studied its effect in Chinese college students.

\section{Methods}

\subsection{Participants}

The participants were college students recruited from Capital Normal University. Volunteers whose C-TMD scores were 37 - 42 points were invited to take part in our research, and most of them told us that mobile phone dependence caused many problems in terms of learning and life, such as sleep, interpersonal communication, consumption, learning efficiency. This study was approved by the Institutional Review Board of the Capital Normal University. To participate, the participants also needed to meet the following criteria, outlined at the pre-workshop evaluation as following: 1) not currently receiving psychotherapy or medication for psychological problems; 2) reading and signing the voluntary consent form. Our final sample contained 42 Chinese college students (11 men and 31 women), with an average age of 19.86 years ( $\mathrm{SD}=1.83)$.

\subsection{Procedure}

The flow of participants through each stage of the study is shown in Figure 1. Forty-two participants were randomized to one of the two conditions: 1) the 


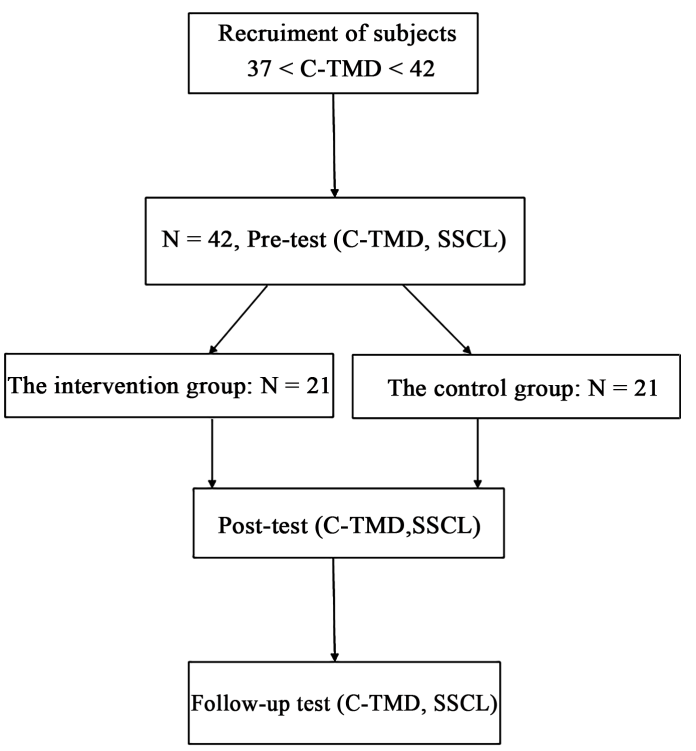

Figure 1. Flow of participants through each stage of the study.

group CBT intervention ( $n=21$ students), or 2$)$ the wait-list control $(n=21$ students). The group CBT intervention included eight weekly, one-hour sessions facilitated by a senior graduate student and co-facilitated by a first-year graduate student in clinical psychology; each group consisted of seven participants. A total of six facilitators conducted the interventions. Students in the wait-list control condition did not receive CBT intervention but finished the follow-up assessment regularly and were then offered the option to participate in the next semester's CBT classes. Self-report measures of C-TMD and SSCL were collected at pre-test, post-test, and two-month follow-up.

All facilitators were master's-level students with at least one year of experience as clinical psychologists. The clinic director who had over 10 years' experience in CBT intervention provided routine clinical supervision for the facilitator. Before the intervention, facilitators were trained in cognitive behavioral therapy for mobile phone dependence. The training included the following sections: 1) Take a group cognitive behavioral therapy course. Learn about techniques related to cognitive behavioral therapy and familiarize yourself with how it is applied in groups. 2) Learn the cognitive behavioral model for mobile phone dependence. Learn to identify the various behaviors, cognition, coexistence problems, and negative consequences related to mobile phone dependency in the model, and learn by analogy. 3) Review the program's eight activities. Study the theory, technology, and activities of each event, and the information to be used for each session. 4) Simulate implementing the group intervention method. As a group, all group leaders and assistants as a group experienced the therapeutic activities.

\section{Group CBT Intervention}

According to the PIU cognitive behavior model defined by Davis (2001), we established a cognitive behavior model for mobile phone dependence (see Figure 2). Dysfunctional behavior consists of the following: 1) forced use of mobile phones, 


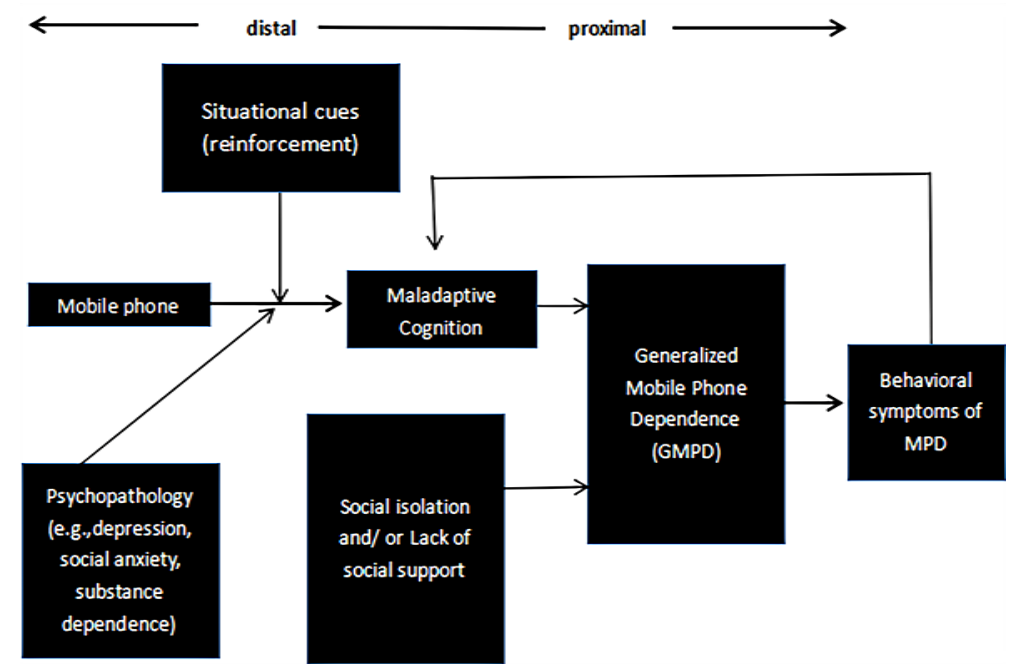

Figure 2. The cognitive-behavioral model of Mobile Phone Dependence (MPD).

even if the individual suffer negative consequences in the work, study, or interpersonal relationships; 2) use of the mobile phone to escape personal problems; 3) leaving the phone causes a withdrawal reaction. Maladaptive cognition refers to the following: 1) obsessive thoughts about the mobile phone; 2) reduced impulse control in mobile phone activities; 3 ) fewer positive feelings about oneself when offline than when online. Previous studies have demonstrated an association among mobile phone dependence, psychological variables (e.g., depression and social isolation), and negative outcomes at work and at home (e.g., Kraut et al., 2002; Young \& Rogers, 1998). The stimuli connecting with mobile phone can result in a conditioned response. The model in Figure 2 suggests that secondary reinforcers act as situational cues that reinforce the development of MPD symptoms and contribute to the maintenance of associated symptoms.

Considering the above-mentioned cognitive behavioral model for mobile dependence and which refers to CBT-IA treatment model, we designed a group CBT for mobile phone dependence, prepared an intervention manual, and conducted an intervention experiment.

Interventions aimed to alleviate an individual's dependence on mobile phones, mainly from the cognitive and behavioral aspects, supplemented by harm reduction therapy to prevent recurrence. In terms of behavior, we first evaluated the participants' current mobile phone usage, then through mobile phone reorganization and the establishment of time management objectives, we helped members measurably change their mobile phone use behaviors. The purpose was to correct mobile phone behaviors and to establish a healthy mobile phone use model. In addition, we would intervene in the members' ability to address issues. In terms of cognition, we helped members identify maladaptive thoughts and understand how they used the phone to escape situations and feelings. Then, the understanding and recognition of cognitive distortions as well as the debate process helped members adjust the bias of reality and maladaptive cognition. Finally, we worked with participants to identify and cope with mem- 
bers of the mobile phone dependence on the development of coexistence factors (personal, situational, social, psychotic or occupational problems) by using harm reduction therapy, thus preventing recurrence. The eight-week intervention included the theme of psychological education, group practice and sharing, and homework, consistent with the basic concepts of cognitive behavior therapy and application. The group program consisted of the following week-by-week plan:

Week 1 Psychoeducation about mobile phone dependence and a motivation feedback form that included a list of consequences for the five major problems caused by the mobile phone dependence and the five possible benefits of controlling phone use, as well as expectation and motivation feedback. The purpose is to establish a good relationship among participants and inspire participation enthusiasm.

Week 2 Identified the predisposing factors in order to develop a corresponding response plan: mobile phone reconstruction, and the establishment of time management objectives for using a mobile phone. The current activity aims to behavioral therapy to reduce mobile phone use.

Week 3 Introduced the concept of automatic ideas and ABCDE theory to help members learn how to identify their own useless automatic ideas, and link automatic ideas with their own emotions. From Week 3 to Week 6, we conduct cognitive therapy.

Week 4 Identified cognitive distortions. Participants learn how to identify cognitive distortions to make change.

Week 5 Reconstructed cognition with challenging cognitive distortions through role-playing techniques. These techniques can help participants to revaluate the reasonability of their belief.

Week 6 Solved the problems that our members experienced after Week 5 when they reconstructed their cognition. Through reconstructing cognition, some members could have trouble in their life. In this week, we will solve these problems together.

Week 7 To prevent recurrence, we combined with the solution from Week 6 to its problem and formed a specific "if ... then ..." action plan to deal with the unknown high predisposing factors.

Week 8 We proposed that every member explore their support system and reviewed previous sessions. The support system can help members to consolidate what they learned.

\subsection{Measures}

Test of Mobile Phone Dependence (TMD; Chóliz, 2012) TMD consists of 22 items measuring three factors: abstinence, lack control/problem, tolerance/interference. The first 10 items ask respondents to use a Likert-type scale ranging from 0 (never) to 4 (frequently); the 12 remaining items are answered on a Likert-type scale from 0 (completely disagree) to 4 (completely agree). Using a revised version of TMD, we studied the psychometric characteristic of 
TMD in 560 Chinese college students. The revised Chinese TMD (C-TMD) consists of 12 items measuring three factors: abstinence, lack control/problem, tolerance/interference. The internal reliability of the C-TMD in this study was good $(\alpha=.79)$. The mean score for this group was $30.34 \pm 7.56$. According to the definition of Internet dependence in the literature (Bai \& Fan, 2005), we defined the mobile phone dependence as the questionnaire scores in the $80 \%-95 \%$ percentile $(37<\mathrm{C}-\mathrm{TMD}<42)$.

Simplified Symptom Checklist (SSCL, Bai \& Fan, 2005) SCL-90 consists of 90 items measuring nine factors. Two subscales of SCL-90 were used, including Obsessive-Compulsive with 10 items and Interpersonal Sensitivity with 9 items (response options: $0=$ no problem to $4=$ very serious). The 19 items were randomly sorted to form a simplified symptom checklist (SSCL) as one of the test scores for the intervention experiment. In this study, the a value for the SSCL was 0.80 , the $\alpha$ value for Obsessive-Compulsive subscale was 0.85 and the $\alpha$ value for Inter personal Sensitivity subscale was 0.82 .

\section{Results}

\subsection{Preliminary Analyses}

Descriptive statistics, including means and standard deviations (SD) for all outcome scores, are presented in Table 1. Participants assigned to the two groups did not differ in demographic characteristics or baseline outcome measures.

\subsection{Analysis Module}

The mixed $2 \times 3$ ANOVA with a repeated-measure over time yielded significant main effects over time for the C-TMD $\left(F[2,80]=14.143, p<.05, \eta^{2}=0.261\right)$ and the $\operatorname{SSCL}\left(F[2,80]=3.454, p<.05, \eta^{2}=0.079\right)$. Significant group main effects were also found for the C-TMD $\left(F[1,40]=31.744, p<.05, \eta^{2}=0.443\right)$ and the

Table 1. Descriptive statistics by intervention groups for study outcome measure.

\begin{tabular}{ccccc}
\hline & \multicolumn{2}{c}{ CB Group } & \multicolumn{2}{c}{ Control Group } \\
\cline { 2 - 5 } & \multicolumn{2}{c}{$n=21$} & \multicolumn{2}{c}{$n=21$} \\
\cline { 2 - 5 } & & $S D$ & $S D$ \\
C-TMD & 39.29 & 2.15 & 38.67 & 1.77 \\
Pre-test & 29.19 & 7.76 & 39.38 & 5.32 \\
Post-test & 28.33 & 7.14 & 38.00 & 6.87 \\
Two-month follow-up & & & & \\
SSCL & 30.76 & 10.65 & 28.48 & 9.33 \\
Pre-test & 23.57 & 10.47 & 28.81 & 13.86 \\
Post-test & 24.95 & 11.94 & 28.95 & 13.18 \\
\hline Two-month follow-up & & & & \\
\hline
\end{tabular}


$\operatorname{SSCL}\left(F[1,40]=0.512, p<.05, \eta^{2}=0.013\right)$. Finally, significant time $\times$ group interactions were detected for the C-TMD $\left(F[2,356]=13.827, p<.05, \eta^{2}=.257\right)$ and the $\operatorname{SSCL}\left(F[2,358]=4.335, p<.05, \eta^{2}=0.098\right)$.

For outcomes with significant time $\times$ group interactions, separate follow-up of repeated-measures ANOVA models tested whether groups showed a significant difference from each other from post-test to two-month follow-up. The mean C-TMD scores for the two groups over time are shown in Figure 3. Post hoc comparisons showed that at pre-test, there was no significant difference between the control group and the intervention group $(p=0.314, d=0.315)$; at post-test the C-TMD scores of the CBT group was significantly lower than that of the control group ( $p<.001, d=1.531)$; at two-month follow-up, the score of the CBT group was significantly lower than that of the control group $(p<.001, d=$ 1.380). The SSCL mean scores for the two groups over time are shown in Figure 4. Post hoc comparisons showed the scores of the intervention group were significantly lower than those of the control group $(p<.05, d=0.427)$ at post-test, and there was marginally significant difference at the two-month follow-up ( $p=$ $0.059, d=0.318)$.

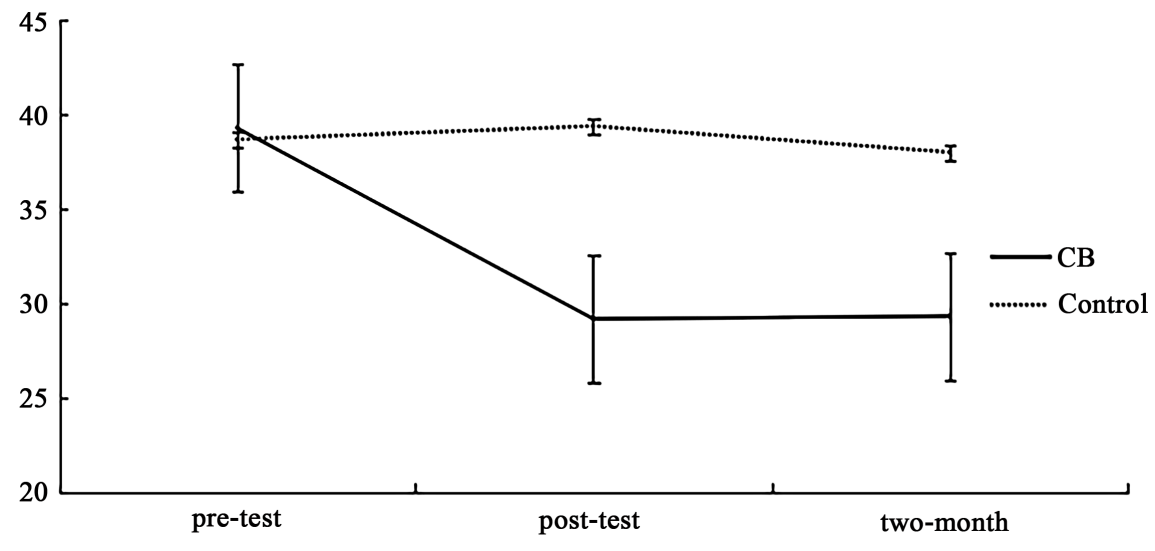

Figure 3. Comparison of C-TMD among two groups at pre-test, post-test, and two-month follow-up.

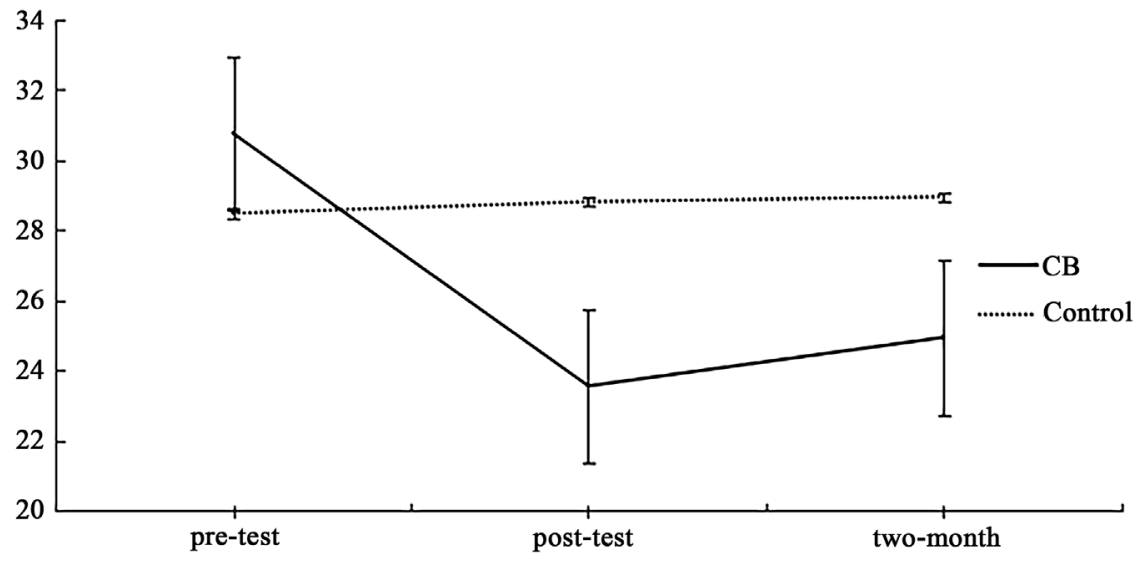

Figure 4. Comparison of SSCL among two groups at pre-test, post-test, and two-month follow-up. 
Separate follow-up repeated-measures ANOVA models tested whether effects over time significantly differed within each group. The mean C-TMD scores for the two groups over time are shown in Figure 3. Multiple post hoc comparisons showed that for CBT group there was a significant difference between the pre-test and post-test $(p<.05)$; the same result was shown between the pre-test and two-month follow-up, but there was no significant difference between the post-test and two-month follow-up. In the control group, there was no significant difference over time. The SSCL mean scores for the two groups over time are shown in Figure 4. Post hoc comparisons showed that in the intervention group, the scores for post-test and two-month follow-up were significantly lower than those for the pre-test $(p<.05)$, but there was no significant difference between post-test and two-month follow-up. In the control group, there was no significant difference over time.

\section{Discussion}

The current study explored whether group CBT would relieve mobile phone dependence. As expected, in comparison with the control group, both the C-TMD scores and SSCL scores among the participants in the intervention group were significantly reduced after training. The difference between pre-training and post-training among participants in the control group was not significant. Furthermore, the intervention group used significantly less mobile phone than did the control group.

First, from the descriptive statistics of the results and the interaction profiles of the time and group, the cell-dependence scores of the intervention group were shown to significantly decrease after 8 weeks, this trend remained 2 months after treatment, and there was no significant difference between the pre-test and post-test for the control group. In the C-TMD, there are questions about the phone usage such as "I used to sleep late or sleep less because I use mobile phone" "I spent more time on the phone, texting (including Wechat), or some APP than I thought". The scores of CBT group on these questions suggested that the frequency of phone usage maintained a downtrend during and after the intervention, but the control group did not, which further demonstrated the possible effect of an eight-week intervention for mobile phone dependence. At the same time, the results also showed that, in the absence of intervention in general, the C-TMD scores of college students generally did not automatically reduce; this suggests that the eight-week CBT designed by this study can be a good way to reduce college students' cell-dependence scores (improve cell phone dependence), and remain effective for 2 months after treatment. As found in previous studies, CBT-IA improves a good effect at improving symptoms associated with Internet addiction after 12 weekly sessions, which persisted one month, three months, and six months after therapy (Young, 2013). As a result, we can hope that CBT might have a long-term effect on mobile phone dependence.

Second, referring to the study of Bai and Fan (2007), we evaluated the intervention experiment by use of the two subscales from the SCL-90 that measure 
compulsive symptoms and interpersonal sensitivity. The results of the study suggested that there was a significant interaction between the group and the time. The results of the simple effect analysis also showed that the score of the intervention group and the control group were significantly different in the posterior measurement, and the difference of the tracing test was marginally significant. This suggests that the study's eight-week CBT can have an effect on the susceptibility of college students to compulsive symptoms and interpersonal relationships, and remains effective for 2 months after treatment, but showing a small recovery trend.

Compulsive symptoms and interpersonal sensitivity have been related to Internet dependence in previous studies (Bai \& Fan, 2005). In this study, the intervention for mobile phone dependence has also been shown to improve the obsessive-compulsive symptoms and interpersonal sensitivity of members. The relationship between obsessive-compulsive symptoms, interpersonal sensitivity, and mobile phone dependence should be investigated in the future research.

Finally, this study evaluated the effect of the intervention through the members, the leader, and the helper's feedback in the form of interviews. The observer was also responsible for documenting the attendance. Twenty of the $21 \mathrm{mem}-$ bers expressed satisfaction to the experimenters; only one said the subjective feeling was not obvious. Three leaders were satisfied with the group intervention plan and the implementation of the evaluation; 3 assistants said that members of the group activities were more cooperative and enthusiastic. In order for the intervention to work, the leaders must understand and be able to identify automatic thinking. In addition, we should pay more attention to the enthusiasm and focus of members.

The study was limited in several ways. First, the sample size of this study was small and the duration of the follow-up study was not long. In the future research, we hope that a larger sample size will provide a higher statistical power. The study's length of tracking should be increased, and survival analysis would be preferable for assessing the effectiveness of the intervention program. Second, the participants were recruited from Capital Normal University, which has more women than men, so there are more women than men in the study. Due to a lack of male participants, we did not study the gender differences in cell phone dependence and the effect of intervention. Chóliz (2012) reported that women use mobile phones more than men, and girls are more likely to engage in mobile phone abuse and experience problems with their parents due to excessive use. We hope to increase the number of male volunteers in future studies to examine gender differences. Furthermore, by studying the relationship between obsessive-compulsive symptoms, interpersonal sensitivity, and mobile phone dependence, we can better understand mobile phone dependence and improve the intervention program. In addition, we can assess whether the mobile phone dependence evolves into mobile addiction in longitudinal studies, and determine the essential differences between the mobile phone dependence and addiction. 
It is worth noting that for a teenager, being dependent on one's phone may not be an addiction but rather a critical method of maintaining social contact. In addition, some young people see internet use, especially gaming, as a welcome escape from the dehumanising pressures (Blaszczynski, 2014). However, when mobile phone use negatively affects their daily life, an effective prevention will be important.

\section{Conclusion}

In conclusion, the eight-week CBT designed in this study can reduce the college students' mobile phone dependence, obsessive-compulsive symptoms and interpersonal sensitivity. The current work also contributes to the prevention of mobile phone addiction. Given the effect of group CBT on the mobile phone dependence, we can promote and apply it in the future.

\section{Acknowledgements}

This research was funded by the National Natural Science Project of China (31571143) \& (31771223).

\section{Funding}

This research was funded by the National Natural Science Project (31571143) \& (31771223).

\section{Conflicts of Interest}

The authors declare no conflicts of interest regarding the publication of this paper.

\section{References}

Aboujaoude, E., Koran, L. M., Gamel, N., Large, M. D., \& Serpe, R. T. (2006). Potential Markers for Problematic Internet Use: A Telephone Survey of 2,513 Adults. CNS Spectrums, 11, 750. https://doi.org/10.1017/S1092852900014875

American Psychiatric Association (2013). Diagnostic and Statistical Manual of Mental Disorders: DSM-V. Diagnostic and Statistical Manual of Mental Disorders. American Psychiatric Association. https://doi.org/10.1176/appi.books.9780890425596

Bai, X., \& Fan, F. M. (2005). A Study on the Internet Dependence of College Students: The Revising and Applying of a Measurement. Psychological Development and Education, 4, 99-104.

Bai, X., \& Fan, F. M. (2007). The Effects of Group Counseling on Internet-Dependent College Students. Chinese Mental Health Journal, 21, 247-250.

Beard, K. W., \& Wolf, E. M. (2001). Modification in the Proposed Diagnostic Criteria for Internet Addiction. CyberPsychology \& Behavior 4, 377-383. https://doi.org/10.1089/109493101300210286

Beck, A. T. (1976). Cognitive Therapy and the Emotional Disorders. New American Library.

Beck, A. T., Wright, F. D., Newman, C. F., \& Liese, B. S. (1993). Cognitive Therapy of 
Substance Abuse. New York: Guilford Press.

Blaszczynski, A. (2014). Youth and Internet Addiction in China. International Gambling Studies, 14, 181-182. https://doi.org/10.1080/14459795.2014.881530

Block, J. J. (2008). Issues for DSM-V: Internet Addiction. American Journal of Psychiatry, 165, 306. https://doi.org/10.1176/appi.ajp.2007.07101556

Bianchi, A., \& Phillips, J. G. (2005). Psychological Predictors of Problem Mobile Phone Use. CyberPsychology \& Behavior, 8, 39-51. https://doi.org/10.1089/cpb.2005.8.39

Billieux, J., Linden, M. V. D., \& Rochat, L. (2008). The Role of Impulsivity in Actual and Problematic Use of the Mobile Phone. Applied Cognitive Psychology, 22, 1195-1210. https://doi.org/10.1002/acp.1429

Brod, C. (1984). Technostress: The Human Cost of the Computer Revolution. In Asia-Pacific Computer and Human Interaction (p. xviii). Washington, DC: IEEE Computer Society.

Chen, L., Yan, Z., Tang, W., Yang, F., Xie, X., \& He, J. (2016). Mobile Phone Addiction Levels and Negative Emotions among Chinese Young Adults. Computers in Human Behavior, 55, 856-866. https://doi.org/10.1016/j.chb.2015.10.030

Chóliz, M. (2012). Mobile-Phone Addiction in Adolescence: The Test of Mobile Phone Dependence (TMD). Progress in Health Sciences, 12, 33-44.

Davis, R. A. (2001). A Cognitive-Behavioral Model of Pathological Internet Use. Computers in Human Behavior, 17, 187-195. https://doi.org/10.1016/S0747-5632(00)00041-8

Hucker, S. J. (1995). Disorders of Impulse Control. Handbook of Forensic Psychology.

Johansson, A., Nordin, S., Heiden, M., \& Sandstrãm, M. (2010). Symptoms, Personality Traits, and Stress in People with Mobile Phone-Related Symptoms and Electromagnetic Hypersensitivity. Journal of Psychosomatic Research, 68, 37-45. https://doi.org/10.1016/j.jpsychores.2009.06.009

Koivusilta, L., Lintonen, T., \& Rimpelä, A. (2003). Mobile Phone Use Has Not Replaced Smoking in Adolescence. BMJ, 326, 161. https://doi.org/10.1136/bmj.326.7381.161

Konok, V., Gigler, D., Bereczky, B. M., \& Miklósi, Á. (2016). Humans’ Attachment to Their Mobile Phones and Its Relationship with Interpersonal Attachment Style. Computers in Human Behavior, 61, 537-547. https://doi.org/10.1016/j.chb.2016.03.062

Korpinen, L. H., \& Pääkkönen, R. J. (2009). Self-Report of Physical Symptoms Associated with Using Mobile Phones and Other Electrical Devices. Bioelectromagnetics, 30, 431. https://doi.org/10.1002/bem.20500

Kraut, R., Kiesler, S., Boneva, B., Cummings, J., Helgeson, V., \& Crawford, A. (2002). Internet Paradox Revisited. Journal of Social Issues, 58, 49-74. https://doi.org/10.1111/1540-4560.00248

Leena, K., Tomi, L., \& Arja, R. (2005). Intensity of Mobile Phone Use and Health Compromising Behaviours-How Is Information and Communication Technology Connected to Health-Related Lifestyle in Adolescence? Journal of Adolescence, 28, 35-47. https://doi.org/10.1016/j.adolescence.2004.05.004

Lepp, A., Barkley, J. E., \& Karpinski, A. C. (2015). The Relationship between Cell Phone Use and Academic Performance in a Sample of US College Students. Sage Open, No. 1, $1-9$.

Lu, X., \& Yeo, K. J. (2015). Pathological Internet Use among Malaysia University Students: Risk Factors and the Role of Cognitive Distortion. Computers in Human Behavior, 45, 235-242. https://doi.org/10.1016/j.chb.2014.12.021

Mei, S., Chai, J., Wang, S. B., Ng, C. H., Ungvari, G. S., \& Xiang, Y. T. (2018). Mobile 
Phone Dependence, Social Support and Impulsivity in Chinese University Students. International Journal of Environmental Research and Public Health, 15, 504. https://doi.org/10.3390/ijerph15030504

Sanchez-Martinez, M., \& Otero, A. (2009). Factors Associated with Cell Phone Use in Adolescents in the Community of Madrid (Spain). Cyberpsychology \& Behavior the Impact of the Internet Multimedia \& Virtual Reality on Behavior \& Society, 12, 131-137.

Shapira, N. A., Lessig, M. C., Goldsmith, T. D., Szabo, S. T., Lazoritz, M., Gold, M. S. et al. (2003). Problematic Internet Use: Proposed Classification and Diagnostic Criteria. Depression \& Anxiety, 17, 207-216. https://doi.org/10.1002/da.10094

Statistics, C. I. (2015). China Internet Network Information Center.

Thomée, S., Eklöf, M., Gustafsson, E., Nilsson, R., \& Hagberg, M. (2007). Prevalence of Perceived Stress, Symptoms of Depression and Sleep Disturbances in Relation to Information and Communication Technology (ICT) Use among Young Adults-An Explorative Prospective Study. Computers in Human Behavior, 23, 1300-1321. https://doi.org/10.1016/j.chb.2004.12.007

Thomée, S., Härenstam, A., \& Hagberg, M. (2011). Mobile Phone Use and Stress, Sleep Disturbances, and Symptoms of Depression among Young Adults-A Prospective Cohort Study. BMC Public Health, 11, 66. https://doi.org/10.1186/1471-2458-11-66

Tian, Y., Bian, Y., Han, P., Gao, F., \& Wang, P. (2017). Associations between Psychosocial Factors and Generalized Pathological Internet Use in Chinese University Students. Computers in Human Behavior, 72, 178-188. https://doi.org/10.1016/j.chb.2017.02.048

Toda, M., Monden, K., Kubo, K., \& Morimoto, K. (2006). Mobile Phone Dependence and Health-Related Lifestyle of University Students. Social Behavior \& Personality: an International Journal, 34, 1277-1284. https://doi.org/10.2224/sbp.2006.34.10.1277

Walsh, S. P., White, K. M., Cox, S., \& Young, R. M. (2011). Keeping in Constant Touch: the Predictors of Young Australians' Mobile Phone Involvement. Computers in $\mathrm{Hu}$ man Behavior, 27, 333-342. https://doi.org/10.1016/j.chb.2010.08.011

Young, K. S. (1998). Internet Addiction: The Emergence of a New Clinical Disorder. $C y$ berpsychology \& Behavior, 1, 237-244. https://doi.org/10.1089/cpb.1998.1.237

Young, K. S. (2013). Treatment Outcomes Using cbt-ia with Internet-Addicted Patients. Journal of Behavioral Addictions, 2, 209. https://doi.org/10.1556/JBA.2.2013.4.3

Young, K. S., \& Rogers, R. C. (1998). The Relationship between Depression and Internet Addiction. Cyberpsychology \& Behavior, 1, 178-183.

https://doi.org/10.1089/cpb.1998.1.25 